\title{
Direct Electron Field
}

National Cancer Institute

\section{Source}

National Cancer Institute. Direct Electron Field. NCI Thesaurus. Code C152054.

A radiation therapy technique where external electron beams are used to deliver treatment to superficial malignancies while sparing underlying organs at risk. 